\title{
RIED. Revista Iberoamericana de Educación a Distancia.
}

\section{Trayectoria 2013-2014}

Lorenzo García Aretio

María García Pérez

Beatriz Tasende Mañá

UNED

Haciéndolo coincidir con los Encuentros Iberoamericanos de la Asociación Iberoamericana de Educación Superior a Distancia, la Revista Iberoamericana de Educación a Distancia (RIED) realiza un balance de los cambios y mejoras acontecidos presentándolos mediante una memoria de actividades que aquí se ofrece como reflejo de la evolución vivida, en este caso, durante los años 2013-14. Años en los que se ha trabajado intensamente para que la RIED cumpla con todos los criterios de calidad que exige la actual situación de las revistas científicas.

Como sucedió en informes anteriores, en este documento se recogen los aspectos de mejora que son ejes transversales, pero a su vez puntos centrales, dentro de los indicadores de calidad. Igualmente seguimos, como es lógico, atendiendo las indicaciones de las agencias de calidad. Vamos a centrar nuestro análisis en estos aspectos destacados:

- Criterios de calidad informativa de la revista como medio de comunicación científica.

- Criterios sobre la calidad del proceso editorial.

- Líneas futuras. Criterios sobre la calidad científica de la revista.

\section{CRITERIOS DE CALIDAD INFORMATIVA DE LA REVISTA COMO MEDIO DE COMUNICACIÓN CIENTÍFICA}

La RIED, Revista Iberoamericana de Educación a Distancia, cumple con prácticamente todos los criterios de calidad informativa que se tienen en cuenta como medio de difusión científica. En este apartado nos detendremos en aquellos que han sido un objetivo primordial para alcanzar las cuotas de calidad más altas. Así, analizaremos una serie de aspectos relevantes que ayudan a esos logros pretendidos. 


\subsection{Apertura exterior del Comité Científico y Evaluadores Externos}

Para cumplir con estos requisitos de máxima calidad y exigencia científica la RIED ha hecho un considerable esfuerzo para reunir en su Comité Científico un elevado número de relevantes estudiosos e investigadores, expertos en los ámbitos propios de la RIED con una gran pluralidad de procedencia institucional y geográfica.

En el momento actual nuestra revista cuenta con un ComitéCientífico conformado por 70 investigadores, expertos internacionales en los ámbitos propios de la RIED. Este grupo viene siendo reforzado en sus tareas de evaluación por una base de datos de otros expertos que vienen desarrollando tareas como Revisores Externos y con perfil cercano al de los miembros del Comité Científico.

Todos los miembros de la red de expertos (Comité Científico y Evaluadores Externos) son ajenos a los Consejos Asesor y Editorial y su función se centra en asesorar y evaluar la publicación, avalándola científicamente y proyectándola internacionalmente. En la siguiente gráfica podemos ver la representación, tanto de los miembros del Comité Científico como de los Evaluadores Externos, que intervinieron como tales en estos dos años, según países de procedencia.

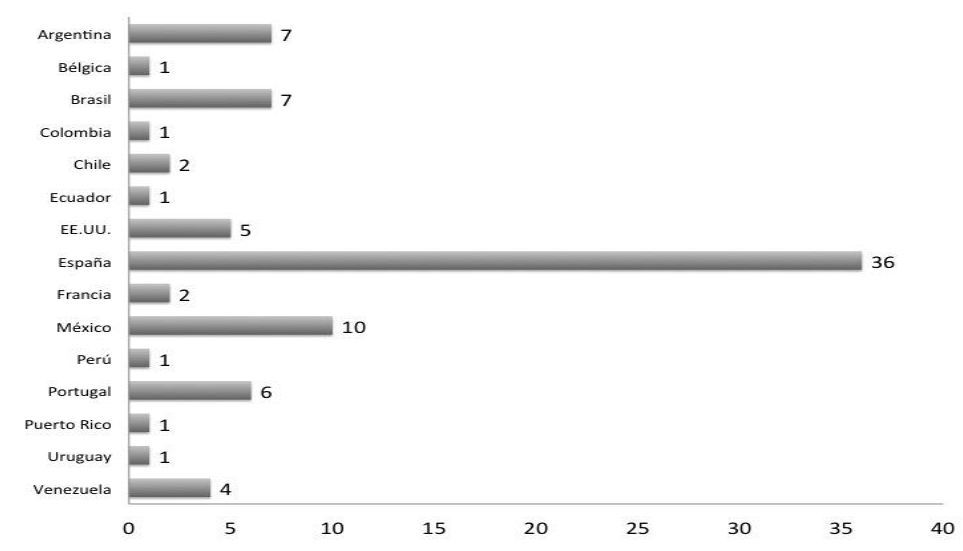

Apertura exterior de los Evaluadores Externos y del Comité Científico

Como se decía en memorias anteriores, y se puede ver en la gráfica siguiente, que un buen número de los Evaluadores Externos y de los miembros del Comité Científico son de nacionalidad española. Sin embargo, queremos resaltar que al tratarse de una revista internacional, necesitamos diversificar el número de los miembros para 
incluir a más expertos investigadores de otras nacionalidades, pretendiendo así el criterio de máxima apertura internacional.

En esta nueva etapa, la RIED se ha dotado de un relevante Consejo Editorial y de Redacción, formado por destacados expertos en el ámbito de la educación a distancia y de las tecnologías aplicadas a la educación. En este Consejo se integran asistentes de dirección, editores de sección, correctores de estilo, responsables de publicación, técnicos expertos en visibilidad y difusión de revistas científicas, documentalistas, maquetadores, etc. Este Consejo Editorial y de Redacción asume funciones vitales para garantizar la calidad científica y editorial de la revista.

\subsection{Contador de visitas de la web como medio de difusión mediática}

Uno de los indicadores que mide la calidad informativa y de difusión de la revista RIED es el contador de visitas situado en su página Web. A través de él podemos comprobar la evolución de la misma, el impacto mediático, y el interés que despierta en la Red. El siguiente gráfico contiene las visitas que ha recibido a partir de los datos arrojados por informes anteriores.

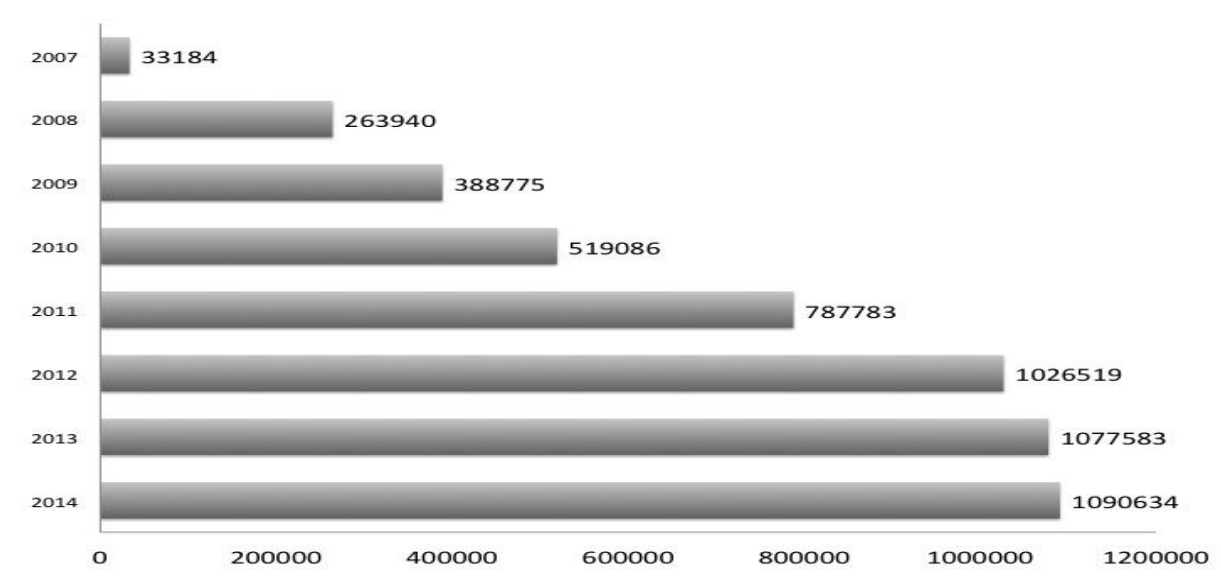

Aumento de las visitas recibidas en la página web de la RIED

Es importante resaltar del anterior gráfico, que la fecha de consulta de accesos en el año 2014 se produjo el 26 de septiembre de 2014. Por otra parte, desde que RIED cuenta con dos sitios Web (años 2013 y 2014), el alojado en la UTPL y el basado en el OJS, alojado en la UNED, solo se ha considerado el contador de UTPL, por lo que los datos totales serán mucho más altos, desde que RIED comparte sitio Web. 
En todo caso, el aumento de visitas a la Web, de RIED solo en UTPL, ha sido muy significativo y de progresión acelerada.

\subsection{Intercambio y suscripciones}

Otra de las líneas que marcan los criterios de calidad en cuanto al impacto mediático de la revista es el apartado de suscripciones e intercambios. Es necesario establecer unos vínculos de intercambios con otras revistas con el fin de ser introducidos en el mayor número de instituciones posibles, por esta razón,este es otro de los objetivos prioritarios sobre los que en este tiempo se ha venido trabajando.

En el año 2012, la RIED tenía establecidos acuerdos de intercambio con un total de 69 revistas de 16 países, destacando 15 revistas de España, 15 de Brasil, 10 de México, 6 de Venezuela y 5 de Argentina. Pues bien, con los datos actuales (septiembre de 2014), la RIED establece acuerdos de intercambio con un total de 81 revistas de 16 países diferentes, entre los que destacan: 18 revistas españolas, 16 de Brasil, 15 de México, 6 de Argentina y 5 de Venezuela.

\subsection{OJS (Open Journal System) para el aumento del impacto mediático}

OJS (Open Journal System) es una herramienta que permite la publicación de los contenidos en abierto, pudiendo ser utilizado únicamente como portal para la difusión de contenidos online. Desde el año 2012, la RIED se embarcó dentro de este sistema como medio de difusión y también como medio de gestión. Actualmente la RIED, además de seguir contando con su sede electrónica en la Universidad Técnica Particular de Loja (UTPL) de Ecuador, reside en la plataforma Open Journal Systems (OJS), una solución de código abierto para la gestión y publicación de revistas académicas en línea (http://e-spacio.uned.es/revistasuned/index.php/ried). Este sistema tiene como objetivo la mejora de la calidad general y el rigor académico de la publicación de revistas especializadas mediante diversas innovaciones, como la mayor transparencia de las políticas que rigen dichas publicaciones y el perfeccionamiento de la indexación.

Por otra parte, una característica muy destacable de OJS consiste en la posibilidad de asignar de manera automática el número DOI a todos los artículos publicados. En la sede electrónica del OJS de la UNED todos los artículos de la RIED, desde su nacimiento en 1998, están ya dados de alta (activados) en el DOI (Digital Object Identifier). Recordemos que el DOI es un sistema que permite identificar cualquier objeto digital en el ciberespacio con independencia de la URL de la que dependa o del servidor que lo soporte. Por este motivo, el DOI se está convirtiendo en uno de los 
elementos de calidad a tener en cuenta en cualquier publicación online de carácter científico.

Así, con el DOI nos podemos valer para citar, referenciar, publicar y buscar artículos y documentos científicos. Otra ventaja del DOI es el poder utilizarlo en artículos pre-print o en prensa (in press). Y ello porque cada vez se hace más perentorio publicar con celeridad los resultados y productos de la investigación de calidad. Por eso se van sumando revistas que proponen una sección de trabajos pre-publicados, (articles in press) es decir, sin volumen y número definitivos. Se trataría de artículos que ya fueron evaluados positivamente y que próximamente serán publicados en formato digital y/o impreso en un volumen/número próximo de la revista. Estos artículos cuentan con el DOI único y exclusivo, por lo que ya pueden ser citados aún a sabiendas de que no existe volumen ni número de adscripción. La RIED en un futuro no lejano se sumará a estos procedimientos.

\subsection{Indización. Inclusión en las Bases de datos}

Durante el año 2013, y parte del año 2014, hemos conseguido, incluir la RIED en diferentes bases de datos, portales, buscadores y catálogos de Alemania, Brasil, España, Chile, Uruguay, México, Argentina, Colombia, Costa Rica y Reino Unido.

A continuación se detalla la indización actual de la RIED dividido según su catalogación:

\begin{tabular}{|c|c|c|}
\hline INDIZACIÓN & $\mathrm{N}^{\mathrm{O}}$ & NOMBRE \\
\hline Bases de datos & 12 & $\begin{array}{l}\text { - BASE (Base de datos de la Biblioteca de la Universidad } \\
\text { de Bielefeld, Alemania). } \\
\text { - CAPES (Coordenação de Aperfeiçoamento de Pessoal } \\
\text { de Nível Superior) } \\
\text { - Cedal (Instituto Latinoamericano de Comunicación } \\
\text { Educativa (ILCE) de México) } \\
\text { - CIRC (Clasificación Integrada de Revistas Científicas) } \\
\text { - CREDI- OEI (Centro de Recursos de la OEI) } \\
\text { - Dialnet (Alertas de Literatura Científica Hispana) } \\
\text { - HEDBIB (International Bibliographic Database on } \\
\text { Higher Education) } \\
\text { - Iresie (Índice de Revistas de Educación Superior e } \\
\text { Investigación Educativa) } \\
\text { - ISOC (CSIC/CINDOC). Consejo Superior de } \\
\text { Investigaciones Científicas de España } \\
\text { - Psicodoc } \\
\text { - Redalyc } \\
\text { Redined }\end{array}$ \\
\hline
\end{tabular}




\begin{tabular}{|c|c|c|}
\hline INDIZACIÓN & $\mathrm{N}^{0}$ & NOMBRE \\
\hline $\begin{array}{l}\text { Plataformas } \\
\text { de evaluación } \\
\text { de revistas }\end{array}$ & 6 & $\begin{array}{l}\text { - CARHUS Plus+, DICE (Difusion y Calidad Editorial } \\
\text { de Revistas), IN-RECS (Índice de Impacto de Revistas } \\
\text { Españolas de Ciencias Sociales), MIAR (Matriz para } \\
\text { Evaluación de Revistas), QUALIS-CAPES, RESH } \\
\text { - Revistas Españolas de Ciencias Sociales (CSIC- } \\
\text { CINDOC) }\end{array}$ \\
\hline $\begin{array}{l}\text { Directorios } \\
\text { selectivos }\end{array}$ & 2 & $\begin{array}{l}\text { - Latindex Catálogo Selectivo (Publicaciones Científicas } \\
\text { Seriadas de América, España y Portugal) } \\
\text { - Ulrich's Periodicals (CSA) }\end{array}$ \\
\hline $\begin{array}{l}\text { Portales y } \\
\text { repositorios } \\
\text { especializados }\end{array}$ & 11 & $\begin{array}{l}\text { - Actualidad Iberoamericana, Asociación Internacional } \\
\text { de Estudios en comunicación social, CLARISE- } \\
\text { Comunidad Latinoamericana Abierta Regional de } \\
\text { Investigación Social y Educativa, Educa.ar, Enlaces } \\
\text { educativos en español de la Universitat de València, } \\
\text { e-sPacio-UNED. Repositorio institucional dela UNED, } \\
\text { Periódicos CAPES, Plataforma de revistas } 360^{\circ} \text {, } \\
\text { Red Iberoamericana de Revistas de Comunicación y } \\
\text { Cultura, REDIAL \& CEISAL, Universia }\end{array}$ \\
\hline $\begin{array}{l}\text { Buscadores } \\
\text { de literatura } \\
\text { científica } \\
\end{array}$ & 6 & $\begin{array}{l}\text { - DOAJ, Dulcinea, Google Académico, Recolecta, } \\
\text { Scirus, Sherpa Romeo }\end{array}$ \\
\hline $\begin{array}{l}\text { Catálogos de } \\
\text { biblioteca }\end{array}$ & 27 & $\begin{array}{l}\text { - Biblioteca de la Universidad Autónoma de Madrid, } \\
\text { Biblioteca de la UC3M, Biblioteca de la Universidad } \\
\text { de Granada, Biblioteca de la Universidad de Huelva } \\
\text { - Biblioteca de la Universidad de Málaga, Biblioteca de } \\
\text { la UNAM, British Library, Buz, Catàleg Col-lectiu de } \\
\text { les Universitats de Catalunya, Catálogo Colectivo de } \\
\text { Publicaciones Periódicas Español CCPP, Catálogo de } \\
\text { la Biblioteca de Educación (Ministerio de Educación, } \\
\text { Cultura y Deporte, Catálogo del CSIC (CIRBIC), } \\
\text { CENDOC, CIDE, COMPLUDOC } \\
\text { - FAMA- Universidad de Sevilla, ICDL, INRP, ICE } \\
\text { (Institute of Education. University of London), KINGS } \\
\text { - MIGUEL DE CERVANTES, REBIUN, UBUCAT, UIB } \\
\text { - WORDLCAT, ZDB }\end{array}$ \\
\hline
\end{tabular}

\section{Indización de la RIED}

Asimismo, después del pertinente período de evaluación, la RIED ha sido aceptada para su inclusión en las bases de datos que detallamos a continuación. 


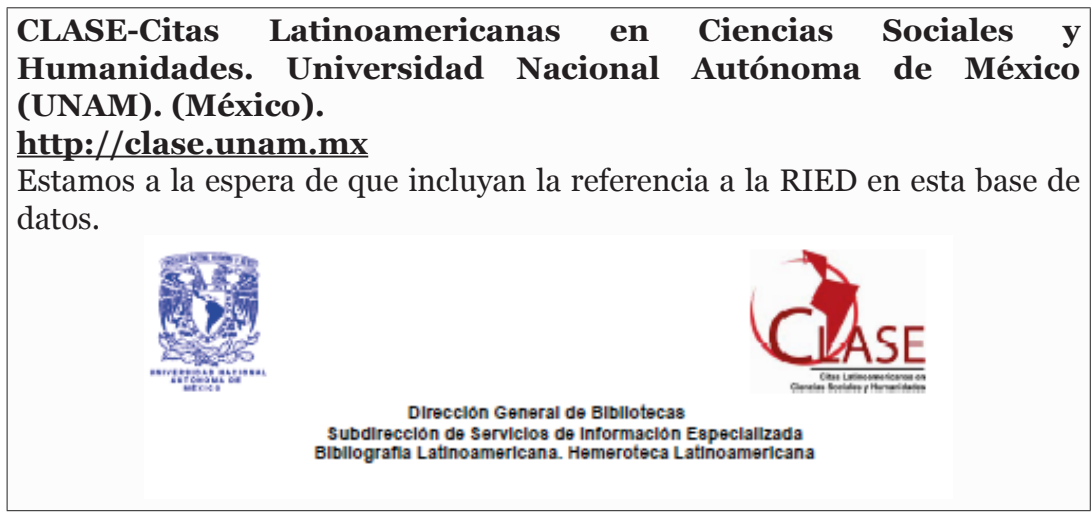

EBSCO Publishing. (Estados Unidos).

http://www.ebscohost.com/

EBSCO nos ofrece la posibilidad de ser nosotros mismos los encargados de subir los números completos y cada uno de los artículos de la RIED a su página web. Para ello nos facilitan las claves de su FTP. Actualmente estamos realizando la subida de todo el contenido de la RIED.

Proceso de indización de la RIED

Además, la RIED se encuentra en proceso de evaluación para ser indizada en:

- Scopus (Holanda): http://www.scopus.com/

- Redalyc (México): http://redalyc.uaemex.mx/

- e-Journals (Suiza): http://www.e-journals.org/

- Hapi (Estados Unidos): http://hapi.ucla.edu/

- ERIH (Noruega): https://www2.esf.org/asp/ERIH/Foreword/search.asp

\subsection{Visibilidad en la Red}

Uno de los mayores retos de las revistas científicas es aumentar su visibilidad para atraer a un mayor público de expertos interesados en el área. Existen diversas estrategias formales e informales para alcanzar dicha visibilidad. Entre las estrategias formales se encuentra la inclusión en diversas bases de datos nacionales e internacionales, como ya era señalado en el apartado anterior. Pero también existen estrategias informales que merecen ser implementadas para alcanzar las cuotas requeridas.

Para lograr la mayor visibilidad en la Red, la RIED ha implementado varios sistemas que proponemos seguidamente. 


\subsubsection{Google Académico}

En el año 2014 hemos abierto el perfil RIED en el más relevante de los buscadores académicos, el Google Scholar. Los datos objetivos de citas realizadas en el mundo académico a los diferentes artículos publicados en la RIED, así como sus índices "h" e "i10" son los siguientes:

CITAS RIED 2009 - octubre 2014

\begin{tabular}{lll}
\hline & Total & 2009 \\
\hline Citas & 1451 & 1090 \\
Índice h & 17 & 16 \\
\hline Índice i10 & 41 & 30 \\
\hline
\end{tabular}

Citas de la RIED en Google Scholar

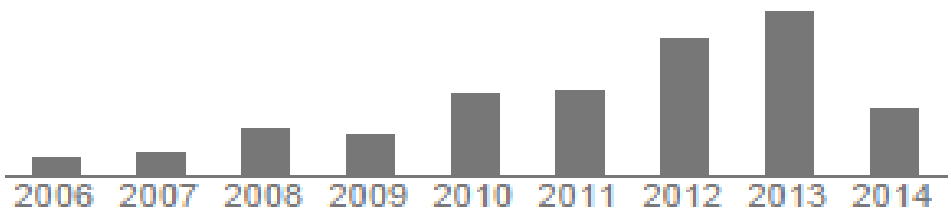

\section{Citas RIED en Google Scholar}

Como puede observarse en las tablas anteriores, la RIED ha obtenido hasta octubre de 2014 un total de 1451 citas, y en los últimos cinco años 1090, siendo el índice "h" de 17 y el índice "i10" de 41. Podría afirmarse que publicar en RIED es garantía de que otros van a leer esos trabajos y, probablemente, a citarlos.

\subsubsection{Blog de la RIED}

El 29 de marzo de 2014 se abre el Blog Oficial de la RIED. Revista Iberoamericana de Educación a Distancia (http://blogderied.blogspot.com.es/), con la intención de difundir las novedades más destacadas en torno a la RIED, así como los artículos relevantes que en la misma se vayan publicando. En el Blog estamos cuidando que la información más esencial sobre RIED esté plenamente actualizada y escrita en tres idiomas: español, portugués e inglés. 


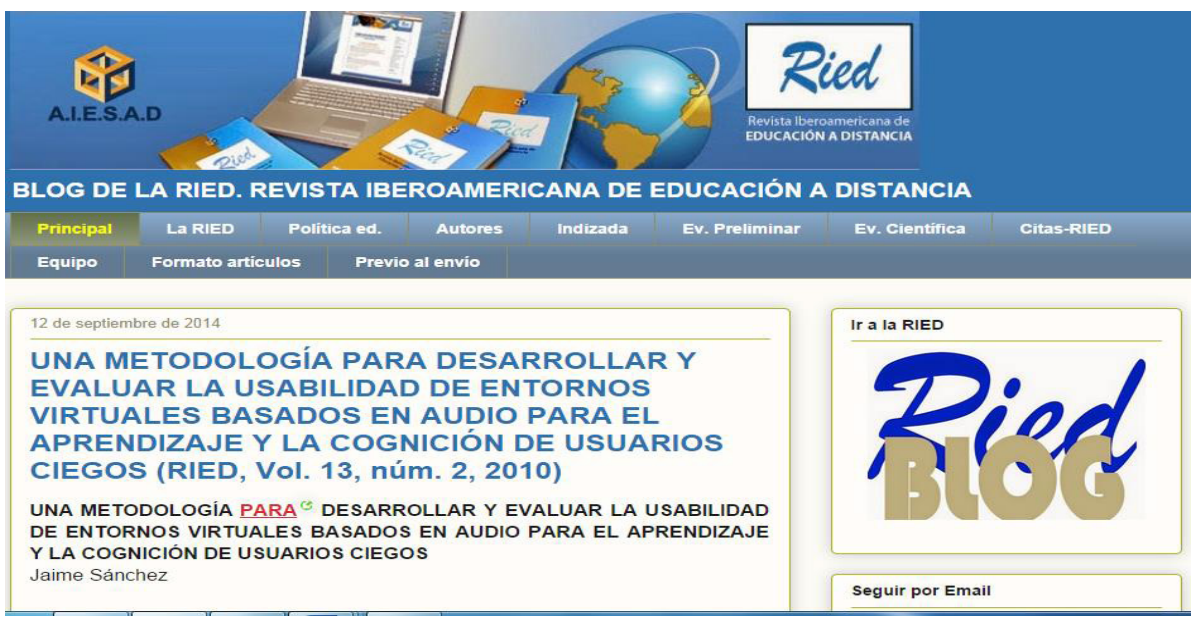

Blog de la RIED

Desde el blog se puede acceder a 10 páginas en las que el lector puede conocer los principios, la misión y objetivos, los órganos y la periodicidad de la RIED; su política editorial; las normas para publicar en RIED; las bases de datos y catálogos en los que se encuentra indizada; los protocolos de evaluación preliminar y de evaluación científica; el índice de citas de la RIED; los miembros del equipo editorial; el formato exigido para los artículos y los requisitos que deben tener en cuenta los investigadores antes de enviar sus trabajos.

Desde su creación se han publicado más de 80 entradas en las que, además de ofrecer puntualmente las novedades de esta nueva etapa de RIED, se van mostrando cíclicamente los resúmenes de los artículos publicados en los últimos años.

Las visitas recibidas desde su creación hasta el 15/10/2014 han sido 12.080.

\subsubsection{La RIED en TWITTER (@revistaRIED)}

Una de las herramientas de comunicación y difusión que la RIED utiliza es la cuenta de Twitter @revistaRIED (https://twitter.com/revistaRIED). Recién creada, cuenta ya con más de 600 seguidores. Desde su creación se han publicado unos 600 tweets sobre artículos de interés de la RIED.

\subsubsection{La RIED en FACEBOOK}

La cuenta de la RIED. Revista Iberoamericana de Educación a Distancia en Facebook (RIED EaD) permite el intercambio de experiencias y opiniones entre docentes e investigadores del ámbito de la Educación a Distancia, especialmente 
de América Latina. Fue creada en abril de 2014. Posteriormente, en septiembre de 2014, se creó una página institucional de RIED en Facebook: https://www.facebook. $\underline{\text { com/EADRied?fref=ts }}$

Las publicaciones realizadas en Facebook suelen ser noticias, informaciones o documentos, de habla hispana, portuguesa o inglesa, cuyos contenidos han sido considerados de especial relevancia para el ámbito de la educación a distancia. Este espacio sirve, a su vez, para que los "amigos" y seguidores de la RIED puedan intercambiar experiencias, comentarios y opiniones sobre las informaciones publicadas y, en general, sobre cualquier aspecto propio de los objetivos de la RIED.

\subsubsection{La RIED en SCOOP.IT}

La RIED. Revista Iberoamericana de Educación a Distancia, utiliza la plataforma de curación de contenidos Scoop.it! (http://www.scoop.it/t/ried-revistaiberoamericana-de-educacion-a-distancia), para la publicación diaria de noticias y artículos de interés relacionados con la educación a distancia.

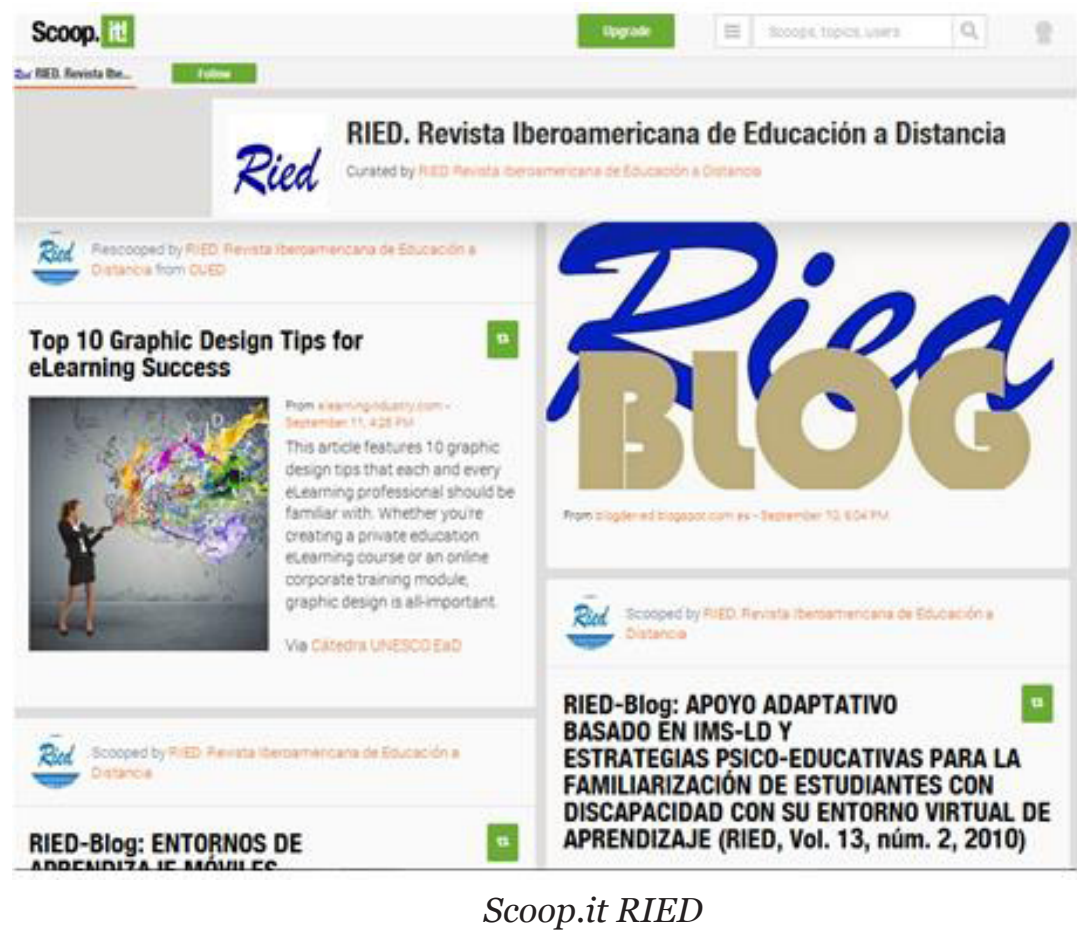

Las publicaciones diarias de esta plataforma se sincronizan con las cuentas de Twitter (@revistaRIED) y Facebook. 


\subsection{Los índices de internacionalidad de la RIED}

EnelDICE(Difusióny Calidad Editorial delas Revistas Españolas deHumanidades y Ciencias Sociales y Jurídicas) la "Valoración de la difusión Internacional" de la RIED es de 7,5 puntos en una escala 0-12 y el índice de "Internacionalidad de sus contribuciones" es "Muy Alta“, 68.52\%.

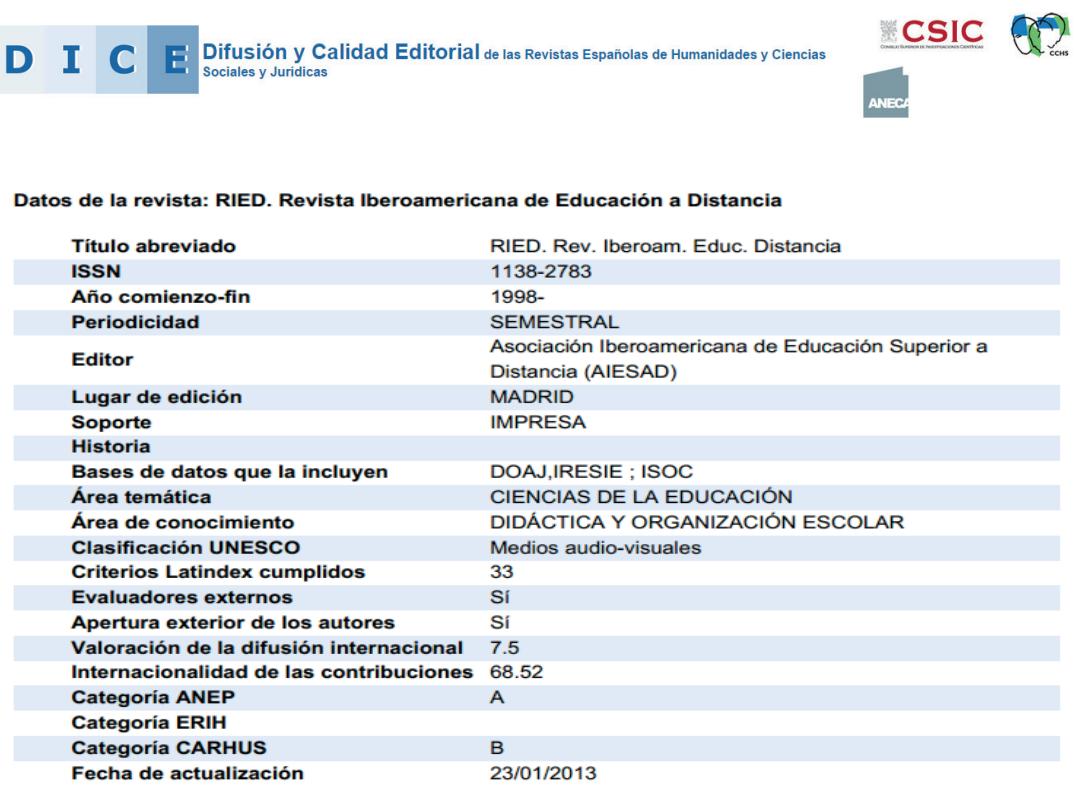

\section{CRITERIOS SOBRE LA CALIDAD DEL PROCESO EDITORIAL}

La calidad del proceso editorial es uno de los puntos principales que es tenido en cuenta en las bases de datos nacionales e internacionales para que una revista sea incluida y valorada, por esta razón este es uno de los factores que se ha consolidado en el tiempo. No obstante, hay que destacar que durante este periodo de tiempo han existido algunos cambios relevantes dentro del proceso editorial para alcanzar una mejora sustancial. En este apartado se van a destacar aquellos cambios que se han producido. 


\subsection{Volúmenes publicados}

La RIED, Revista Iberoamericana de Educación a Distancia, ha afianzado, especialmente en los últimos años, los indicadores relevantes dentro de la calidad editorial siguiendo los parámetros de las bases de datos nacionales e internacionales. Así, la RIED mantiene su edición de números con rigurosa periodicidad ajustándose permanentemente a las fechas de publicación.

No obstante, este último año se ha producido un cambio en el número de artículos que se publican dentro de cada volumen. Uno de los criterios de calidad que se ha tenido en cuenta, por exigencia de alguna base de datos, es el número de artículos publicados, ya que era necesario al menos publicar un mínimo de 20 artículos al año para poder ser incluida en la misma. Este hecho ha conllevado que aunque mantengamos el mismo número de volúmenes, el número de artículos publicados dentro de cada uno de ellos haya variado. Por esta razón la RIED, para cumplir con este requisito, ha publicado desde el anterior informe, el siguiente número de artículos por volumen:

- 16.2 (julio de 2013). Número de artículos por volumen: 9

- 17.1 (enero de 2014). Número de artículos por volumen: 9

- 17.2 (julio de 2014). Número de artículos por volumen: 12

A partir de estos datos se debe señalar que los trabajos recibidos durante este último año ascienden a un total de 81. Este número de artículos se distribuye de la siguiente manera:

- 30 artículos han sido publicados en los volúmenes 16.2, 17.1, y 17.2 obteniendo, por tanto, una evaluación positiva por parte de los miembros del Comité Científico y Evaluadores Externos. Aunque en muchos casos se ha requerido de los autores modificaciones de mejora más o menos sustanciales.

- 14 trabajos más han sido valorados positivamente y están a la espera de ser publicado en siguientes volúmenes.

- 1 está aún en proceso de revisión externa por haberse recibido posteriormente.

- 33 artículos han sido rechazados y no serán publicados en la revista RIED.

Según estos datos, el porcentaje de los artículos aceptados, publicados y rechazados que se ha recibido en la Secretaría de la RIED es el siguiente: 


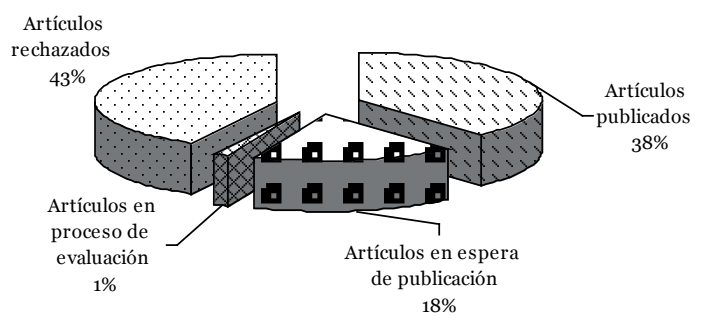

Porcentajes de artículos recibidos

Un aspecto que es importante destacar es la apertura y difusión de la RIED dentro de la comunidad científica, tal y como señalábamos en el apartado anterior. En este sentido, en este último año se ha procedido a ampliar los idiomas de publicación que hasta el momento eran tan solo el español y el portugués, pero debido a las exigencias de las bases de datos internacionales, donde requerían que pudieran existir artículos publicados también en inglés, desde el volumen 17.1 (enero de 2014) se aceptan artículos escritos en dicho idioma.

Pero además, una de las formas de conocer la difusión de nuestra revista es el análisis de los colaboradores que publican o desean publicar en la misma. Así en la siguiente gráfica podremos observar los países de procedencia de los artículos que se han publicado en los dos últimos volúmenes que han sido editados en este último año:

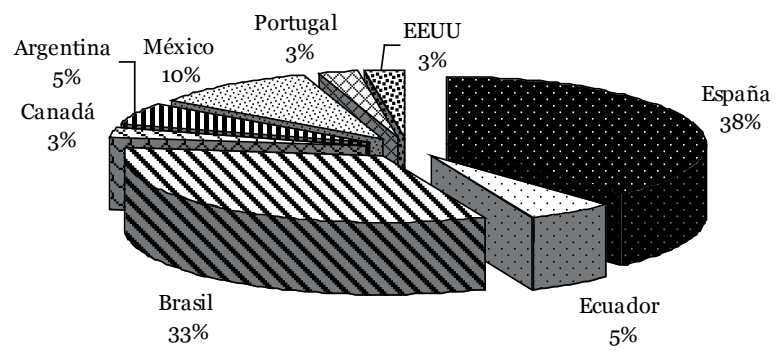

Porcentajes de países de procedencia de artículos publicados. 


\subsection{OJS (Open Journal System) como proceso de gestión editorial}

Como se decía anteriormente, el OJS es un software de código abierto desarrollado por el Public Knowledge Project (PKP) de Canadá, organismo dedicado al desarrollo y aprovechamiento de nuevas tecnologías para la investigación académica y para el acceso universal a la producción científica.

Se trata de un sistema de administración y publicación de revistas científicas y académicas en Internet diseñado para mejorar la gestión de las publicaciones seriadas. Permite un manejo eficiente y unificado del proceso editorial y facilita el acceso al conocimiento producido por universidades y centros de investigación. En los últimos años, OJS se está consolidando como la principal herramienta para el acceso a texto completo de los documentos publicados en revistas científicas de cualquier parte del mundo.

Mediante el OJS las revistas pueden integrar y organizar todas las tareas de gestión y administración a través de un mismo espacio virtual y con una misma herramienta. Por eso, a través de la plataforma de OJS que la UNED ha puesto a disposición de la RIED, nuestra revista se encuentra inserta ya en este sistema.

La principal característica y ventaja de OJS consiste en que permite integrar y organizar todas las tareas de gestión y administración de una publicación electrónica en un mismo espacio virtual y con una misma herramienta. Esto significa que todo el proceso llevado a cabo para la publicación de cualquier revista científica -el envío de los artículos por parte de los autores, la comunicación con cualquier miembro de la comunidad de la revista, la gestión de los originales para su posterior evaluación, el proceso de revisión y corrección, etc.- se ejecuta, siempre y en todo momento, a través del sistema OJS.

El autor que decide enviar un artículo a la RIED debe saber que ese artículo va a pasar por diferentes fases y filtros: recepción, revisión de los aspectos formales y adecuación a los requerimientos de la revista, revisión antiplagio y evaluación por pares expertos referente a los contenidos científicos y metodológicos del artículo. $\mathrm{Si}$ en el artículo existiesen defectos formales, devolución del mismo al autor para que rectifique. Si el artículo pudiera publicarse pero condicionado a modificaciones más o menos sustanciales, devolución al autor, igualmente, para que modifique. Nueva revisión de lo modificado, corrección de estilo, etc. Una vez que el artículo cuenta con todas las valoraciones positivas, se procede a su maquetación. Antes de ser publicado, nueva revisión antiplagio o autoplagio (el artículo podría haber sido publicado por otra revista sin que el autor -faltando a un principio ético- lo hubiera comunicado). Finalmente, si es el caso, al artículo se le asigna un DOI (Digital Object Identifier) y es publicado. 
OJS posee unos roles de usuario encargados de las diversas tareas en el proceso editorial, desde el ingreso de artículos hasta su publicación, todo esto siguiendo un proceso lógico de flujo de trabajo. De esta forma, cada perfil de usuario tiene asignado un nivel de administración específico, desde el perfil del lector hasta el de administrador general.

Son ya numerosas las revistas científicas en todo el mundo, y de la mayoría de los ámbitos de conocimiento, que están utilizando OJS para la publicación y gestión de sus publicaciones electrónicas. En la actualidad, la RIED ya cuenta con su propio espacio OJS completamente diseñado y configurado para una gestión eficaz.

\section{CRITERIOS SOBRE LA CALIDAD CIENTÍFICA DE LA REVISTA}

A partir de este momento, tenemos que marcarnos como referencia otras líneas que hemos puesto en marcha durante este último año y que ya se están llevando a cabo.

- Contenido editorial: Para que la RIED sea incluida en las bases de datos internacionales de mayor prestigio, como es el caso de WoS (JCR) o Scopus, es necesario que aporte investigaciones y estudios que enriquezcan esta base de datos. Por esta razón es vital que los trabajos que se publiquen tengan gran calidad científica y susceptibles de ser citados. Para ello se están realizando frecuentes convocatorias públicas de proyectos para temas monográficos de la revista.

- Diversidad internacional de autores: En los últimos volúmenes ya se ha tenido en cuenta este aspecto, sin embargo, si nos remitimos a los trabajos recibidos la gran mayoría de ellos corresponden aún a colaboradores españoles. Para aumentar dicha visibilidad, los idiomas de publicación se han aumentado, incluyéndose el inglés como idioma de publicación dentro de las normas de la RIED, para dar una mayor cabida a todos los expertos interesados en el campo de la educación a distancia y para ser incluidos en nuevas bases de datos internacionales.

- Análisis de citas: Las bases de datos, como ISI, evalúan que las revistas sean citadas dentro de su campo. Esto es un sinónimo de la difusión de la revista, pero también de la calidad de los trabajos que se publican en ella. En este sentido, en la actualidad en cada uno de los artículos que aparecen en la Web de la RIED se ha añadido la referencia de los mismos para que puedan ser citados con mayor facilidad.

En este informe se han recogido los numerosos cambios que se han producido en la Revista Iberoamericana de Educación a Distancia (RIED) durante los dos últimos años, sin embargo, es imprescindible que los progresos que se han llevado a 
cabo se consoliden y se amplíe nuestra difusión a través de la inclusión de la RIED en más bases de datos nacionales e internacionales, por ello, es nuestro objetivo centrarnos en reforzar e implementar al completo el sistema OJS. 